\title{
Volumetric measurement of dentoalveolar defects by means of intraoral 3D scanner and gravimetric model
}

\author{
Martin J. R. Lindström ${ }^{1} \cdot$ Marianne Ahmad ${ }^{1} \cdot$ Ryo Jimbo $^{1} \cdot$ Arman Ameri $^{2} \cdot$ Per Vult Von Steyern $^{3} \cdot$ Jonas P. Becktor $^{1} \mathbb{C}$
}

Received: 26 October 2017 / Accepted: 10 December 2018 / Published online: 7 January 2019

(c) The Author(s) 2019

\begin{abstract}
The aim of this study was to evaluate the accuracy in volumetric measurements obtained on an experimental model using an intraoral scanner and a gravimetric method. Three identical partial dentate maxillary acrylic models with three fabricated alveolar defects, in anterior and posterior regions, were scanned using an intraoral scanner (20 scans/defects). The defects differed in terms of size and distance of neighbouring teeth. As references, replicas of each defect were created using a dimensional stable silicone impression material. After measuring the mass of each replica, the volume was calculated by dividing the mass of each replica by the density of the impression material. The defects had a volume, according to the gravimetric method, ranging from 40.5 to $143.7 \mathrm{~mm}^{3}$. The scans were imported to metrology software for analyses. Accuracy was determined in terms of trueness and precision. The mean trueness for all defect types was $0.168 \mathrm{~mm}^{3}$ (SD 0.691 , range 2.82). There was no statistical significant difference between the mean trueness for all defects measured $(p=0.910)$. The mean precision for all defect types was $0.147 \mathrm{~mm}^{3}$ (SD 0.524, range 2.86). There were no statistical significant differences between the dental models in regard to mean precision $(p=0.401)$, however, there were statistical significant differences between defects in position 1 and $2(p=0.002)$ and 1 and $3(p=0.001)$. Based on the findings of this study, the intraoral scanner utilized in the current study presented an acceptable level of accuracy when measuring volume of defects.
\end{abstract}

Keywords Surgical techniques $\cdot$ Wound healing $\cdot$ Volumetric measurement $\cdot$ Tissue alterations $\cdot$ Intraoral scanning

\section{Introduction}

Surgical interventions in oral and maxillofacial surgery often engage both soft and hard tissue and they often result in tissue alteration. The interventions may include all type of surgical traumas that generate physiological responses, such as extraction, tissue augmentation and implant treatment, important to understand in the prediction and evaluation of clinical treatment outcome. Bone remodelling is one of the central mechanisms behind the dimensional changes observed after surgical trauma, influencing volumetric

Jonas P. Becktor

jonas.becktor@mah.se

1 Department of Oral and Maxillofacial Surgery and Oral Medicine, Faculty of Odontology, Malmö University, Carl Gustafs Väg 34, 21421 Malmö, Sweden

2 Department of Prosthodontics, Faculty of Odontology, Malmö University, Malmö, Sweden

3 Department of Materials Science and Technology, Faculty of Odontology, Malmö University, Malmö, Sweden dimensions of the bone tissue as well as the soft tissue topography. The ability to monitor and accurately measure volumetric changes in tissues is an important instrument of great value for the clinical evaluation and follow-up of different surgical procedures.

Various methods have been described for the measurement of soft and hard tissue alterations, including conventional X-rays [1], computer tomography (CT) [2], stereoscopic imaging [3], optical scanning [4-11], direct measurements intraorally or on casts $[2,12]$, or mapping by ultrasonic assessments $[13,14]$. However, there are only limited data available regarding the accuracy of these techniques in vivo. Further, the techniques involving ionizing radiation may be questioned due to their invasiveness and the need for radiation exposure. These disadvantages may be compensated by the use of other techniques such as optical scanning and clinical photographs. However, a problem with techniques using images is the superimposing and matching in one common coordinate system, which is critical to enable precise measurements. In addition, the intraoral 3D scanner (IOS) itself has expected technology-related errors 
to a certain level, due to a large number of pieces of measured data that are linked together to profile a 3D object, resulting in distortion of the created data.

The quality of measurements is therefore defined by terms of accuracy. Accuracy itself is the combination of two elements, both important and complementary: "trueness" and "precision". Trueness refers to the ability of a measurement to match the actual value of the object being measured, and that little or nothing deviates from reality. To detect the trueness from IOS, it is mandatory to have a reference model with error tending to zero. Although trueness is the key element for an IOS, it is not sufficient, as it must be accompanied by precision. Precision is defined as the ability of a measurement to be consistently repeated, ensuring that multiple measurements of the same object must necessarily be comparable and differ from each other as little as possible. To measure the precision of an IOS, no reference models are needed: it is sufficient to superimpose different intraoral scans, and evaluate to what extent they deviate, using a dedicated software.

In recent years, the development of IOS in the field of dentistry has resulted in numerous advancements. The IOS comprises the first component in the CAD/CAM system, which captures data of anatomical structures with the use of technologies such as confocal laser scanning, confocal microscopy, active triangulation, wavefront sampling or optical coherent tomography $[15,16]$.

Several studies have evaluated the accuracy of IOS [17-19]. Flügge et al. evaluated the precision of the iTero (Cadent), TRIOS (3Shape) and True Definition (3M ESPE) by the digitization of scanbodies. They found that the precision of the IOS decreased with an increasing distance between the scanbodies when compared to a dental lab scanner (D250, 3Shape) [17].

Previous studies by Müller et al. [20] investigated the impact of different scanning strategies of the TRIOS (3Shape) IOS. They demonstrated that the precision of the scans was lowered when the scanning started with covering the buccal surfaces of the teeth in a full dental arch. Contrary, there was a higher degree of precision when the scanning started with covering the palatal surfaces or in a back and forth manner [20]. These findings demonstrated that the scanning technique itself also seems to have an impact on the accuracy of the IOS.

The IOS system provides a level of accuracy that may be beneficial in analysing the alterations before and after surgical interventions. This technique would provide an additional, less invasive modality to evaluate the outcome of surgical procedures. However, to our knowledge, no previous studies have examined the accuracy of volumetric measurements using an IOS. In such setting, there is a need of a volume sensitive model to use as reference. In the field of physics, volume is commonly calculated by gravimetric analysis with very high accuracy [21]. Thus, it would be of significant interest to test the accuracy of a commercially available device to determine whether it can be utilized for such volumetric evaluation. Hence, the aim of this study was to evaluate the accuracy of an IOS in terms of volume measurements.

\section{Materials and methods}

\section{Models}

Three identical acrylic models of a partial dentate maxilla were used. The models were prepared identically with a defect on the alveolar ridge in the region of tooth number 15, 11 and 25 (referred to as position 1, 2 and 3) (Fig. 1). Each defect differed in size and distance between neighbouring teeth depending on the position of the defect, further presented in (Fig. 2).

\section{Intraoral scanner}

The Trios $3^{\circledR}$ Cart scanner used in this study is the thirdgeneration IOS fabricated by 3-Shape (Copenhagen, Denmark). It works under the principle of confocal microscopy and optical scanning. Trios $3^{\circledR}$ is a fast structured light scanner. It is powder-free and it produces in-colour images. The acquisition software of Trios $3^{\circledR}$ produces proprietary files (.DCM) which can be exported to an open file format (.STL) file by the use of an export plugin provided by the manufacturer.

\section{Gravimetric method}

The physical volume of the defects was determined by creating a replica of each defect using the silicone impression material Imprint ${ }^{\mathrm{TM}} 4$ Bite (3M ESPE). The volume of the replica was quantified by the use of Archimedes' principle, by weighing the impression and dividing the mass of each sample with the specific density of set Imprint ${ }^{\mathrm{TM}} 4$ Bite [21].

Imprint ${ }^{\mathrm{TM}} 4$ Bite was injected into each defect and left to set in accordance with the recommendations from the manufacturer. While setting, the impression material was contoured using a silicone key pre-fabricated in Putty ${ }^{\mathrm{TM}}$ (3M ESPE). Once set, the impression material was removed from the defect. Two impression samples were produced per defect. The samples were placed on a precision weighing balance with a readability of $0.001 \mathrm{~g}$ (Voyager ${ }^{\circledR}$ Pro, Ohaus Corporation, Pine Brook, USA) and the average weight of the two impression samples was taken as the weight of the impression sample for that given defect. The average weight of each impression sample was divided by the known density (1654 gm/mL) for set Imprint TM4 Bite, which was 
Position 1
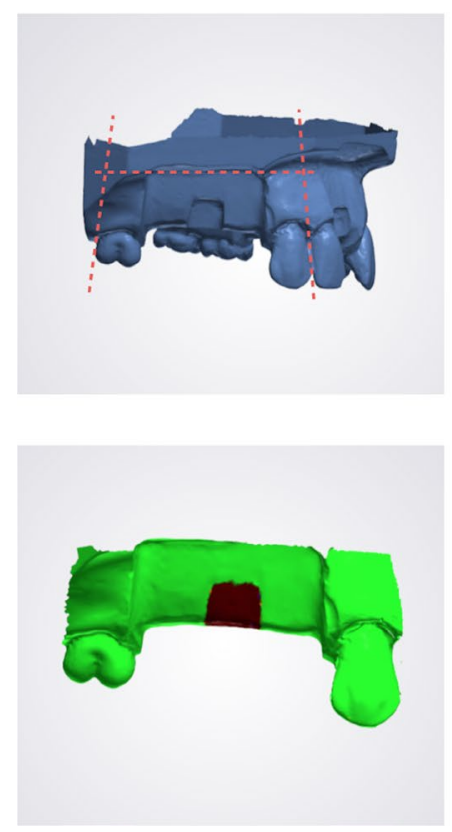

Position 2
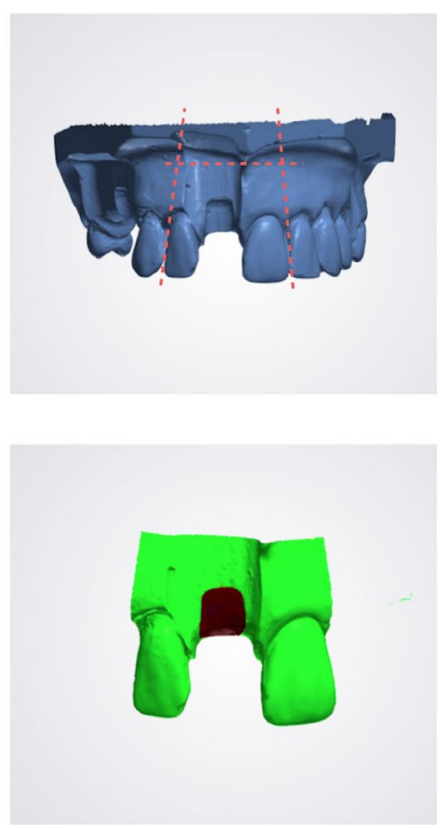

Position 3
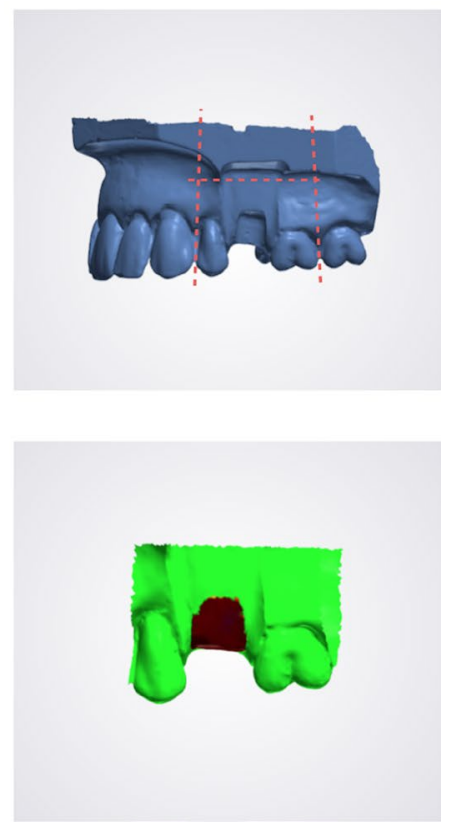

Fig. 1 Aligned scans. 3D scan of acrylic model with alveolar defects in position 1 (tooth 15), position 2 (tooth 11) and position 3 (tooth 25). The volume of each defect was evaluated following superimposing and cropping using $3 \mathrm{D}$ processing software

Fig. 2 Acrylic model. The distance between neighbouring teeth in the acrylic model varied depending on the defect position
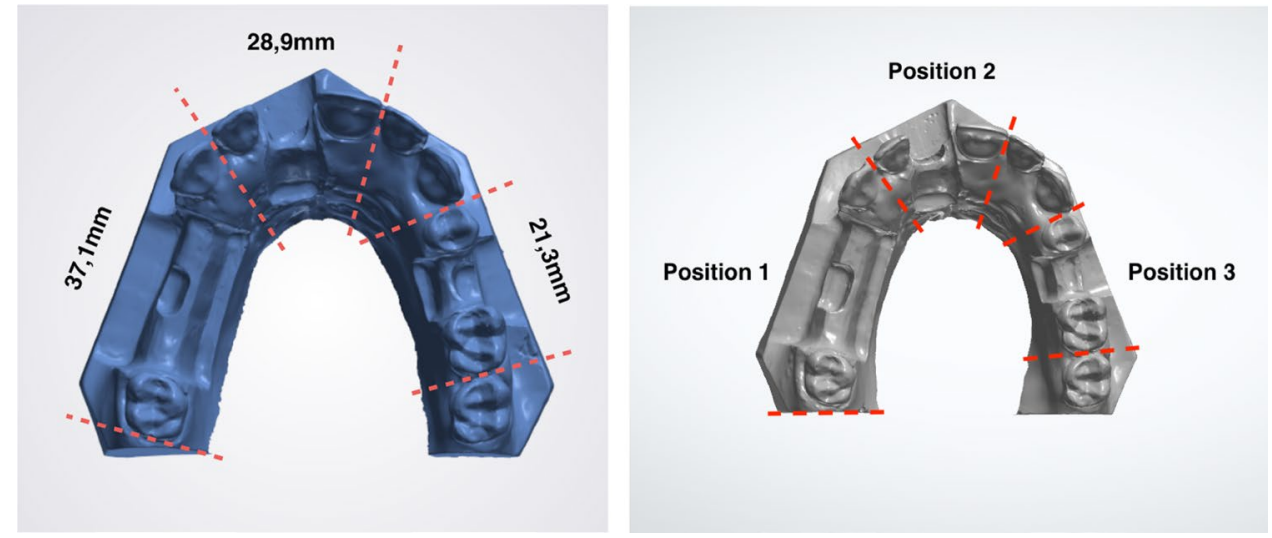

established by the Technical Research Institute of Sweden. This calculation provided the reference volume for each of the defects.

\section{Study protocol}

The test volumes were created by scanning of each defect with neighbouring teeth as reference points (one tooth on each side of the defect). Each defect was scanned 20 times by the IOS, following a previous session of learning scans, to avoid any inaccuracies due to learning curve. The scanning procedure of each defect was made with and without the replica. All the STL datasets from the IOS were loaded into 3D evaluation software Geomagic Qualify ${ }^{\mathrm{TM}}$
2012 (Geomagic, Morrisville, USA). Each scan pair was aligned by superimposition in one common coordinate system using the best fit algorithm to generate the digital volume of the defects. To ensure a precise superimposition, unnecessary information were removed using the "cut with planes" function to trim the digital models as uniform as possible.

\section{Trueness and precision}

For the trueness measurements, the digital datasets of the volume obtained from the scanner were compared to the physical reference volume from the replica of each defect. 
For the precision measurements, the digital datasets of volume obtained from the scanner were compared in terms of deviation.

Only absolute values were used for the analysis, resulting in one value per two scans, in total 120 values.

\section{Statistical analyses}

To detect differences regarding the trueness and precision of volume measurements, a univariate analysis of variance with a full factorial design was fitted. Further, Tukey test and post hoc analysis was implemented. The level of statistical significance was set to $<0.05$. Least-square means were calculated with a $95 \%$ confidence interval. Box plots were used to illustrate experiment results. All calculations were performed using the statistical software SPSS Statistics (IBM Corp. IBM SPSS for Windows, Version 22.0. Armonk, NY: IBM Corp).

\section{Results}

Mean values for trueness and precision are divided in regard to defect position. The univariate analysis with post hoc multiple comparison for observed means, revealed no statistical significant differences between models, stating there were no difference in regard to trueness nor precision dependent on defect size. However, statistical differences were observed regarding precision and defect position.

\section{Trueness}

The mean trueness was $0.168 \mathrm{~mm}^{3}$ (SD 0.691, range 2.82). For defects in position 1 , the mean trueness was $0.236 \mathrm{~mm}^{3}$ (SD 0.708, range 2.32), position $2,0.151 \mathrm{~mm}^{3}$ (SD 0.688, range 2.24 ) and position $3,0.104 \mathrm{~mm}^{3}$ (SD 0.745, range 2.40) (Fig. 3). There was no statistical significant difference between the mean trueness for defects in positions 1,2 or $3(p=0.910)$.

\section{Precision}

The mean precision was $0.147 \mathrm{~mm}^{3}$ (SD 0.524, range 2.86). For defects in position 1 , the mean precision was 0.118 $\mathrm{mm}^{3}$ (SD 0.525, range 1.89), in position $2,0.202 \mathrm{~mm}^{3}$ (SD 0.391 , range 1.55 ) and position $3,0.356 \mathrm{~mm}^{3}$ (SD 0.542, range 1.91) (Fig. 4). There were no statistical significant differences between the different dental models, $(p=0.401)$. Analysis of deviations regarding mean differences dependent on defect position yielded statistical significant differences between defect positions 1 and $2(p=0.002), 1$ and 3 $(p=0.001)$, but not between 2 and $3(p=0.200)$ The greatest deviation in mean difference was observed between defect positions 1 and 3, $0.473 \mathrm{~mm}$.
Fig. 3 Trueness. Box plot of mean trueness of defects in positions 1,2 and 3

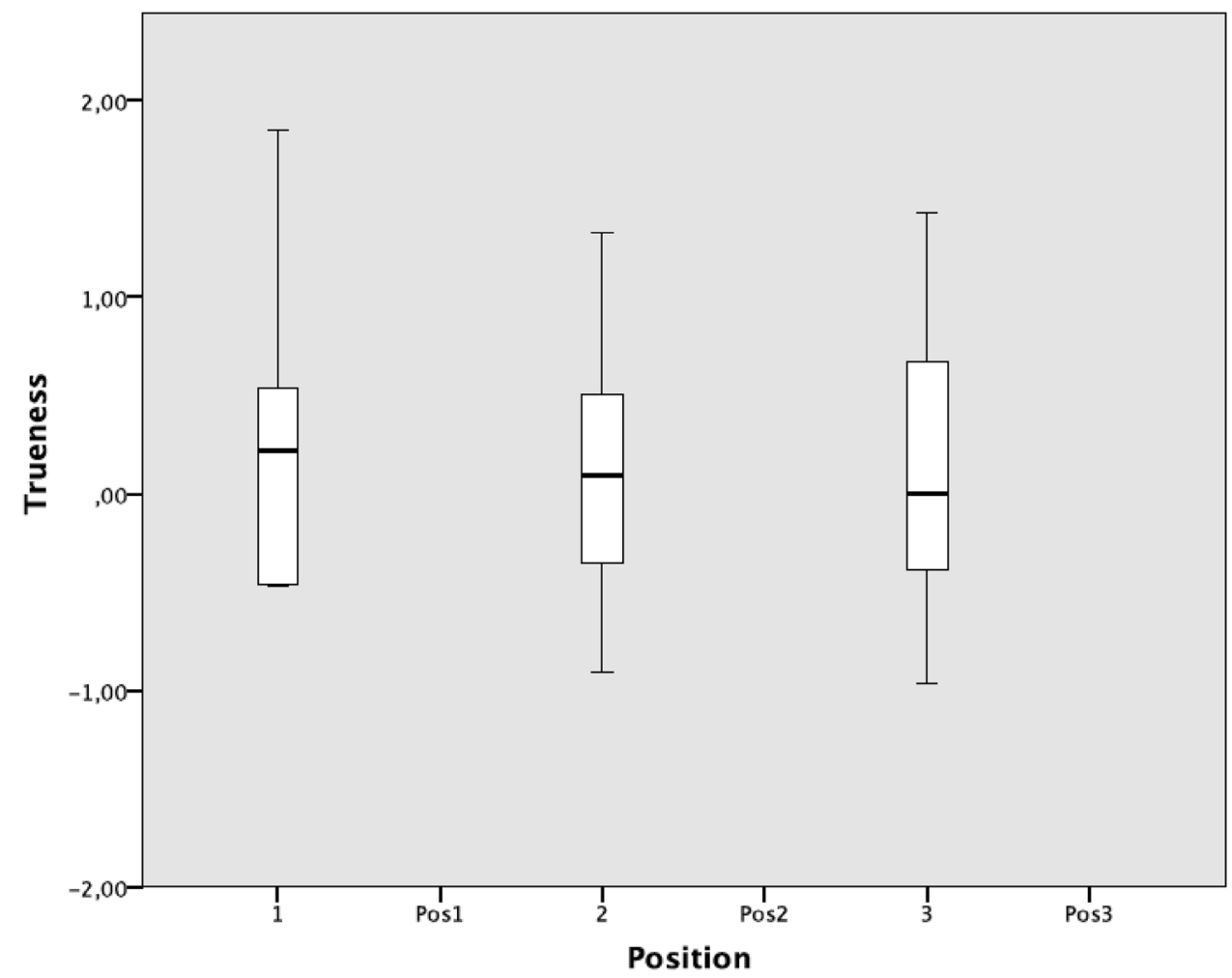


Fig. 4 Precision. Box plot of mean precision of defects in positions 1,2 and 3

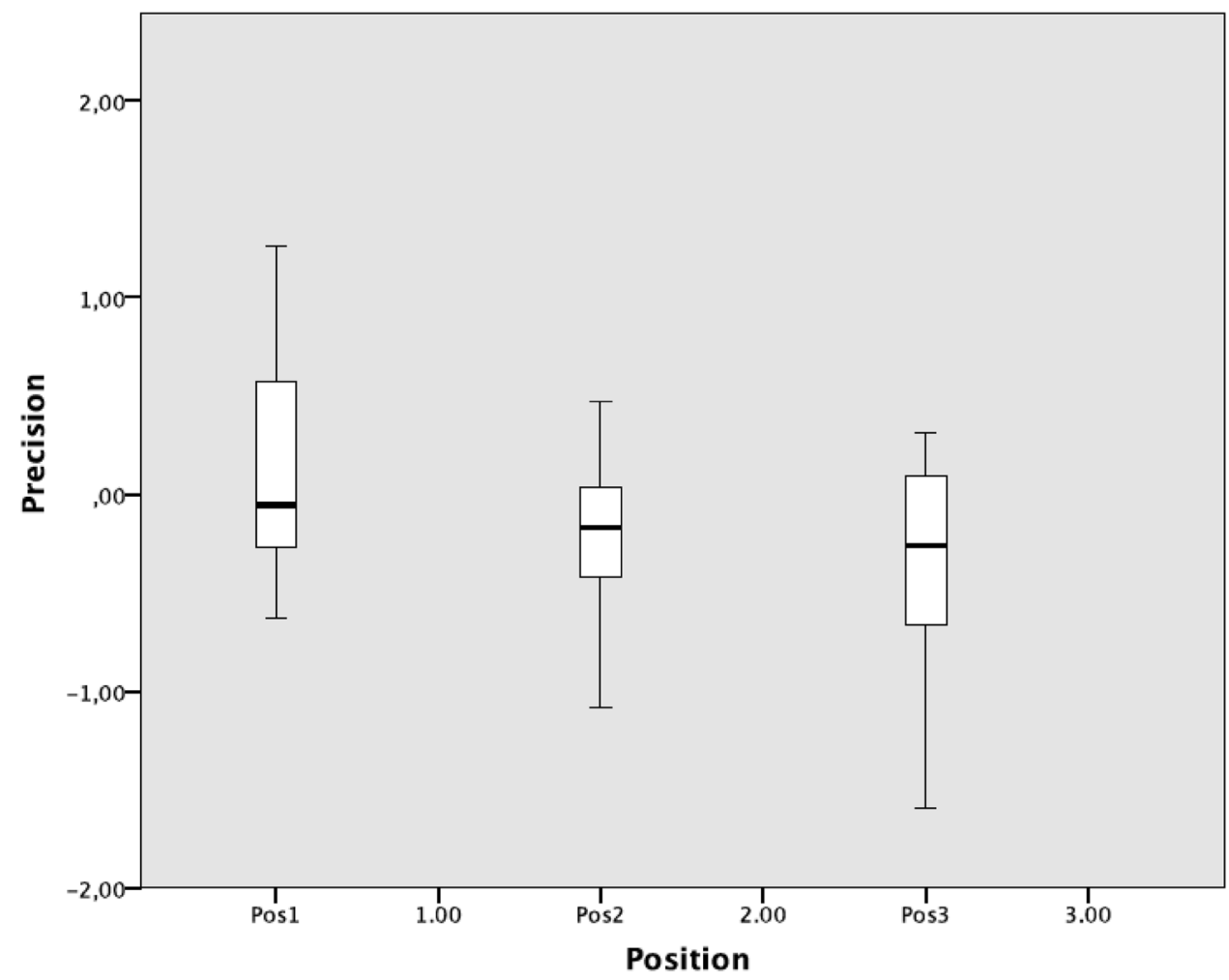

\section{Discussion}

Volumetric analysis of oral tissues has evolved from rough visual inspection to more advanced techniques including cone-beam CT and recently the development of optical scanning technologies. CBCT is a frequently used modality with high accuracy in the surgical evaluation of hard tissue outcome. However, in the post-operative situation with an implant in vicinity of the region of interest, the measurement of data becomes complex due to metallic reflections [22]. The 3D optical scanning technique has mainly been applied to alter conventional dental impressions and facilitate the $\mathrm{CAD} / \mathrm{CAM}$ process in prosthodontic treatment and orthodontic planning. But at a research level, the technique has fairly gained more attention as a method to provide volumetric measurements for evaluating soft and hard tissue alterations after surgical interventions [7, 23, 24]. Further abilities to assemble data from different types of 3D devices (IOS files and CT files) have further improved the so-called digital workflow [25], creating new possibilities such as treatment with guided implant surgery. The use of IOS for volumetric measurement is a simple and non-invasive tool that can provide 3D display making the volumetric analysis only limited to the accuracy of the IOS system.

The volumetric measurements of the present investigation suggested that the TRIOS intraoral scanner possessed high levels of accuracy. However, it was also demonstrated that there was a statistical significant difference between the positions of each defect in terms of precision. This is speculated to be mainly due to different anatomic morphologies and various scan area sizes. It has been reported that the individual patient anatomy is important to take into consideration prior to intraoral scanning. It can be said that depending on the site in the oral cavity, the degree of concavity and convexity can vary and the precision can be influenced. The influence of scan size has been reported by Flügge et al. [17] that an increased scanning area lowered the degree of precision using an intra-oral scanner $[17,18]$. This may be the reason for the minor differences observed in the current study, however, it must also be stated that the observed difference might not have an impact in a clinical setting when comparing volumetric differences.

Several studies have utilized a 3D scanning technique to investigate the volume alterations following different surgical interventions [7, 10, 11, 23, 26-28]. Fickl et al. [7, 27] evaluated the dimensional changes in alveolar bone following tooth extractions in a dog mandible. In the studies from 2008, the volume difference was presented in millimetres following a 2D section of a 3D model, for instance, in a bucco/lingual section. In the latter study in 2009, the volume change was presented as the measured volume difference per measured area. Hence, the extent of volume change is not possible to compare with the defects in the present study. The change of volume alteration during soft tissue augmentation was analysed in a dog study with the help of a 3D scanning technique by Thoma et al. [8]. However, the volumetric changes were measured after soft 
tissue augmentation with different collagen-based matrices in the dog oral cavity, where they presented the volume change per measured area in millimetre. The presented outcome was the same as in two later studies, where they investigated the volume alteration of peri-implant tissues after tooth extraction by Schneider et al. [10, 11]. This did not reveal the total volume of each area of interest, which complicates any comparisons with regard to volume of defects used in the present study.

Gonzalez-Martin et al. [24] investigated the volume alteration following sub-epithelial connective tissue grafting. They used a soft tissue graft, which was harvested pre-prosthetically from the palate of each subject and placed in a sub-epithelial manner on the buccal side in patients lacking a central or lateral incisor. Conventional impressions were taken before and after the surgical treatment, which was latter quantified using a laboratory $3 \mathrm{D}$ scanner. They found a mean volume increase of $35.9 \mathrm{~mm}^{3}$ with a range of $12.8-52.6 \mathrm{~mm}^{3}$ [24]. One can hypothesize that the deviations of volumetric measurements by the intraoral scanner in our study are still within what is a clinically acceptable level of inaccuracy.

Our present study has certain limitations. Foremost, it is an in vitro study. Therefore, the important results obtained here should be necessarily translated into the clinical setting, and validated in vivo, where there are additional factors that can degrade the quality of a scan (saliva, blood, accessibility to the surface of interest and movement of the patient).

Second, although the gravimetric method is a powerful method to calculate volume, it is highly dependent on the choice of material, its ability to flow and fill the volume of interest and its stability upon removal. The use of reference scanners such as optical desktop scanners or MicroCT are highly accurate alternative reference models for measurement of volume and surfaces. Although, it must be remembered that these instruments are limited to in vitro and ex vivo studies and thus are unavailable in a clinical setting. In conclusion, further studies at a clinical level are needed to confirm the outcomes of the present work, and to provide even more interesting data to clinicians.

Acknowledgements The authors acknowledge Peter Fleckenstein for his assistance in regard to the volumetric measurements in $3 \mathrm{D}$.

\section{Compliance with ethical standards}

Conflict of interest The authors declare that they have no conflict of interest.

Open Access This article is distributed under the terms of the Creative Commons Attribution 4.0 International License (http://creativeco mmons.org/licenses/by/4.0/), which permits unrestricted use, distribution, and reproduction in any medium, provided you give appropriate credit to the original author(s) and the source, provide a link to the Creative Commons license, and indicate if changes were made.

\section{References}

1. Alpiste-Illueca F. Dimensions of the dentogingival unit in maxillary anterior teeth: a new exploration technique (parallel profile radiograph). Int J Periodontics Restor Dent. 2004;24(4):386-96.

2. Chen LC, Lundgren T, Hallstrom H, Cherel F. Comparison of different methods of assessing alveolar ridge dimensions prior to dental implant placement. J Periodontol. 2008;79(3):401-5.

3. Jemt T, Book K, Lie A, Borjesson T. Mucosal topography around implants in edentulous upper jaws. Photogrammetric three-dimensional measurements of the effect of replacement of a removable prosthesis with a fixed prosthesis. Clin Oral Implants Res. 1994;5(4):220-8.

4. Jemt T, Lekholm U. Measurements of buccal tissue volumes at single-implant restorations after local bone grafting in maxillas: a 3-year clinical prospective study case series. Clin Implant Dent Relat Res. 2003;5(2):63-70.

5. Jemt T, Lekholm U. Single implants and buccal bone grafts in the anterior maxilla: measurements of buccal crestal contours in a 6-year prospective clinical study. Clin Implant Dent Relat Res. 2005;7(3): 127-35.

6. Walivaara DA, Isaksson S, Rosen S. Description of a technique for evaluation of three-dimensional shape alterations in soft tissue after intra oral bone reconstruction. Br J Oral maxillofac Surg. 2007;45(5):382-6.

7. Fickl S, Schneider D, Zuhr O, Hinze M, Ender A, Jung RE, Hurzeler MB. Dimensional changes of the ridge contour after socket preservation and buccal overbuilding: an animal study. J Clin Periodontol. 2009;36(5):442-8.

8. Thoma DS, Jung RE, Schneider D, Cochran DL, Ender A, Jones AA, Gorlach C, Uebersax L, Graf-Hausner U, Hammerle $\mathrm{CH}$. Soft tissue volume augmentation by the use of collagenbased matrices: a volumetric analysis. J Clin Periodontol. 2010;37(7):659-66.

9. van der Meer WJ, Dijkstra PU, Visser A, Vissink A, Ren Y. Reliability and validity of measurements of facial swelling with a stereophotogrammetry optical three-dimensional scanner. Br J Oral Maxillofac Surg. 2014;52(10):922-7.

10. Schneider D, Grunder U, Ender A, Hammerle CH, Jung RE. Volume gain and stability of peri-implant tissue following bone and soft tissue augmentation: 1-year results from a prospective cohort study. Clin Oral Implants Res. 2011;22(1):28-37.

11. Schneider D, Schmidlin PR, Philipp A, Annen BM, Ronay V, Hammerle CH, Attin T, Jung RE. Labial soft tissue volume evaluation of different techniques for ridge preservation after tooth extraction: a randomized controlled clinical trial. J Clin Periodontol. 2014;41(6):612-7.

12. Cardaropoli G, Lekholm U, Wennstrom JL. Tissue alterations at implant-supported single-tooth replacements: a 1-year prospective clinical study. Clin Oral Implants Res. 2006;17(2):165-71.

13. Eghbali A, De Bruyn H, Cosyn J, Kerckaert I, Van Hoof T. Ultrasonic assessment of mucosal thickness around implants: validity, reproducibility, and stability of connective tissue grafts at the buccal aspect. Clin Implant Dent Relat Res. 2016;18(1):51-61.

14. Chan HL, Wang HL, Fowlkes JB, Giannobile WV, Kripfgans OD. Non-ionizing real-time ultrasonography in implant and oral surgery: a feasibility study. Clin Oral Implants Res. 2017;28(3):341-7.

15. Logozzo S, Zanetti EM, Franceschini G, Kilpelä A, Mäkynen A. Recent advances in dental optics-Part I: 3D intraoral scanners for restorative dentistry. Opt Lasers Eng. 2014;54:203-21.

16. Zimmermann M, Mehl A, Mörmann W, Reich S. Intraoral scanning systems-a current overview. Int J Comput Dent. 2015;18(2):101-29. 
17. Flugge TV, Att W, Metzger MC, Nelson K. Precision of dental implant digitization using intraoral scanners. Int J Prosthodont. 2016;29(3):277-83.

18. Imburgia M, Logozzo S, Hauschild U, Veronesi G, Mangano C, Mangano FG. Accuracy of four intraoral scanners in oral implantology: a comparative in vitro study. BMC Oral Health 2017;17(1):92.

19. Hack GD, Patzelt SB. Evaluation of the accuracy of six intraoral scanning devices: an in-vitro investigation. ADA Prof Prod Rev. 2015;10(4):1-5.

20. Muller P, Ender A, Joda T, Katsoulis J. Impact of digital intraoral scan strategies on the impression accuracy using the TRIOS Pod scanner. Quintessence Int (Berlin, Germany, 1985) 2016;47(4):343-9.

21. Ahlowalia MS, Patel S, Anwar HM, Cama G, Austin RS, Wilson $\mathrm{R}$, Mannocci F. Accuracy of CBCT for volumetric measurement of simulated periapical lesions. Int Endod J. 2013;46(6):538-46.

22. Razavi T, Palmer RM, Davies J, Wilson R, Palmer PJ. Accuracy of measuring the cortical bone thickness adjacent to dental implants using cone beam computed tomography. Clin Oral Implants Res. 2010;21(7):718-25.

23. Fickl S, Zuhr O, Wachtel H, Stappert CF, Stein JM, Hurzeler MB. Dimensional changes of the alveolar ridge contour after different socket preservation techniques. J Clinical Periodontol. 2008;35(10):906-13.

24. Gonzalez-Martin O, Veltri M, Moraguez O, Belser UC. Quantitative three-dimensional methodology to assess volumetric and profilometric outcome of subepithelial connective tissue grafting at pontic sites: a prospective pilot study. Int J Periodontics Restor Dent. 2014;34(5):673-9.

25. Hara S, Mitsugi M, Kanno T, Tatemoto Y. Computer-aided design provisionalization and implant insertion combined with optical scanning of plaster casts and computed tomography data. Ann Maxillofac Surg. 2014;4(1):64-9.

26. Fickl S, Zuhr O, Wachtel H, Bolz W, Huerzeler M. Tissue alterations after tooth extraction with and without surgical trauma: a volumetric study in the beagle dog. J Clin Periodontol. 2008;35(4):356-63.

27. Fickl S, Zuhr O, Wachtel H, Bolz W, Huerzeler MB. Hard tissue alterations after socket preservation: an experimental study in the beagle dog. Clin Oral Implants Res. 2008;19(11):1111-8.

28. Lione R, Franchi L, Huanca Ghislanzoni LT, Primozic J, Buongiorno M, Cozza P. Palatal surface and volume in mouth-breathing subjects evaluated with three-dimensional analysis of digital dental casts—a controlled study. Eur J Orthod. 2015;37(1):101-4.

Publisher's Note Springer Nature remains neutral with regard to jurisdictional claims in published maps and institutional affiliations. 